R. J. Cohen and W. T. Sullivan, III, eds.

\title{
Observations of Artificial Space Objects in Lviv Astronomical Observatory
}

\author{
Jeva Vovchyk, Jaroslav Blagodyr and Olexandr Logvinenko \\ Astronomical Observatory of Lviv State University, 8 Kyryla and \\ Mefodij st., 290005, Lviv, Ukraine, e-mail eve@astro.franko.Lviv.ua
}

\begin{abstract}
Space debris is the price which humankind must pay for entering space. And as this debris is dangerous in different ways, there must be ways to identify, catalogue, and predict the positions of, these cosmic bodies. That is why it is necessary to observe all artificial cosmic objects that are in space. One way of observing debris is by photometry. Photometric observations give light-curves from which one may deduce information about the position in space, the form, size, and other parameters of the object. Since 1975 a research group at Lviv Astronomical Observatory has been working at the problem of recording light-curves of different artificial cosmic objects. Four electrophotometers were constructed and developed. Many light curves of different artificial objects were measured with these electrophotometers. All light curves are collected in the local computer data bank and are available for use by anybody who needs such information.
\end{abstract}

\section{Introduction}

Space debris includes all those artificial objects which are flying in space but never again will be useful for humankind (Flury 1991). These objects are dangerous to spacecraft, they adversely affect our atmosphere and they give misleading information to astronomical observatories. That is why it is so important to monitor space debris.

There are four different ways to observe any artificial satellite: radio, optical location, position, and photometric observations. The information received can be collected in a databank of artificial satellites (such as exists in the Institute of Astronomy Russian Science Academy or in NASA). As a rule such databanks are based on the orbital parameters of the objects (which are obtained from position measurements). However the orbital information is not always sufficient to identify the object, and then other types of observation are needed. For example, information about the shape and size of the object could be obtained from photometric observations.

\section{Photometers for Observing Artificial Satellites}

Since 1975 a research group at Lviv Astronomical Observatory has worked on the construction of electrophotometers for observing artificial satellites (Blagodyr at 


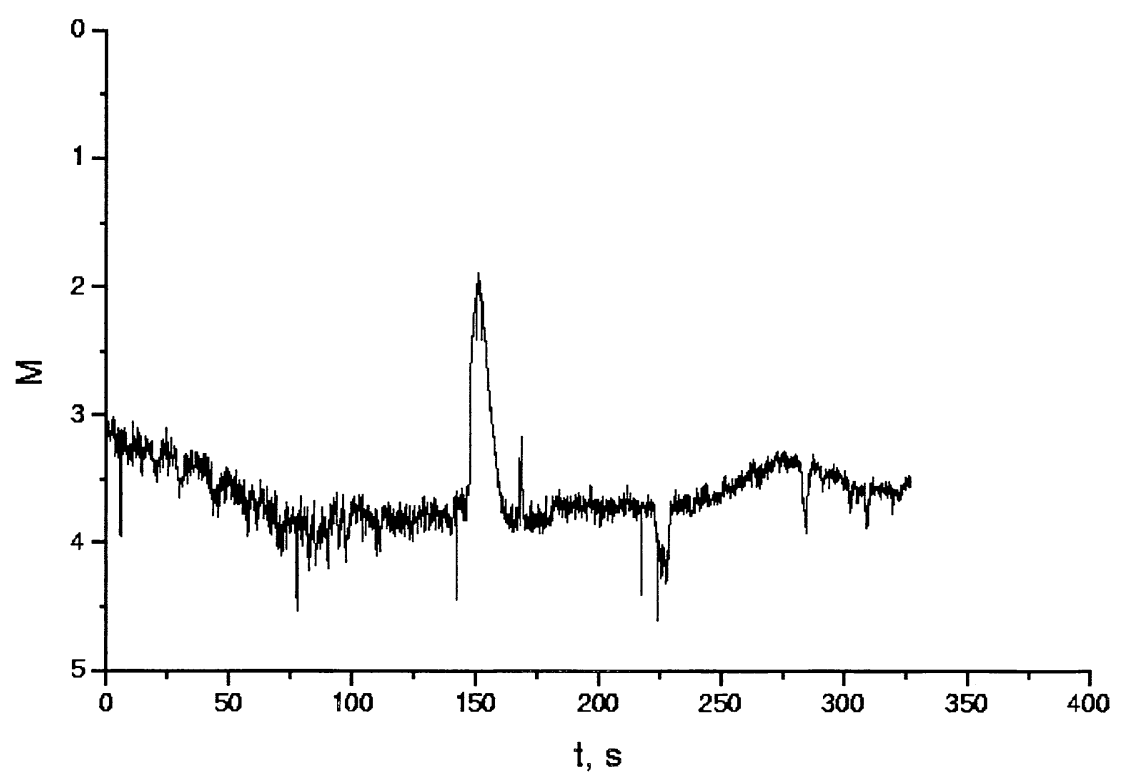

Figure 1. The light-curve of the TOPEX measured on 10th May 1999. The zero of time is at $22 \mathrm{~h} 13 \mathrm{~m} 26,34 \mathrm{~s}$ UT.

all, 1996). A family of four electrophotometers have been constructed. Each of the instruments has a different number of optical channels (one, two, four and/or eight) and different filters. Observations can be made in integrated light or in the BV system.

The multichannel instruments are mounted on four-axis astronomical mountings and have $10-\mathrm{cm}$ objectives. That gives the posibility to observe objects in low Earth-orbit (distances from some hundreds to some thousands of kilometers). The single-channel photometer is mounted on the telescope with twinaxis equatorial mounting and is intended for observing artificial satellites in the geostationary orbit - the distance to these objects is $36000 \mathrm{~km}$.

In all instruments the resulting signals are recorded by computer in "realtime" and all instruments have time-channels with 0.01-s time accuracy.

\section{Results of Observations}

Many light curves of different artificial satellites were measured with these electrophotometers. All light curves are collected in the local computer databank and are available for use by anybody who needs such information.

Examples of the results are shown in Figures 1 and 2. Fig.1 shows the light-curve of the object TOPEX-POSEIDON received on the 10th May 1999. The begining of the light-curve (zero on the time axis) is at $22 \mathrm{~h} 13 \mathrm{~m} 26,34 \mathrm{~s}$ UT. 


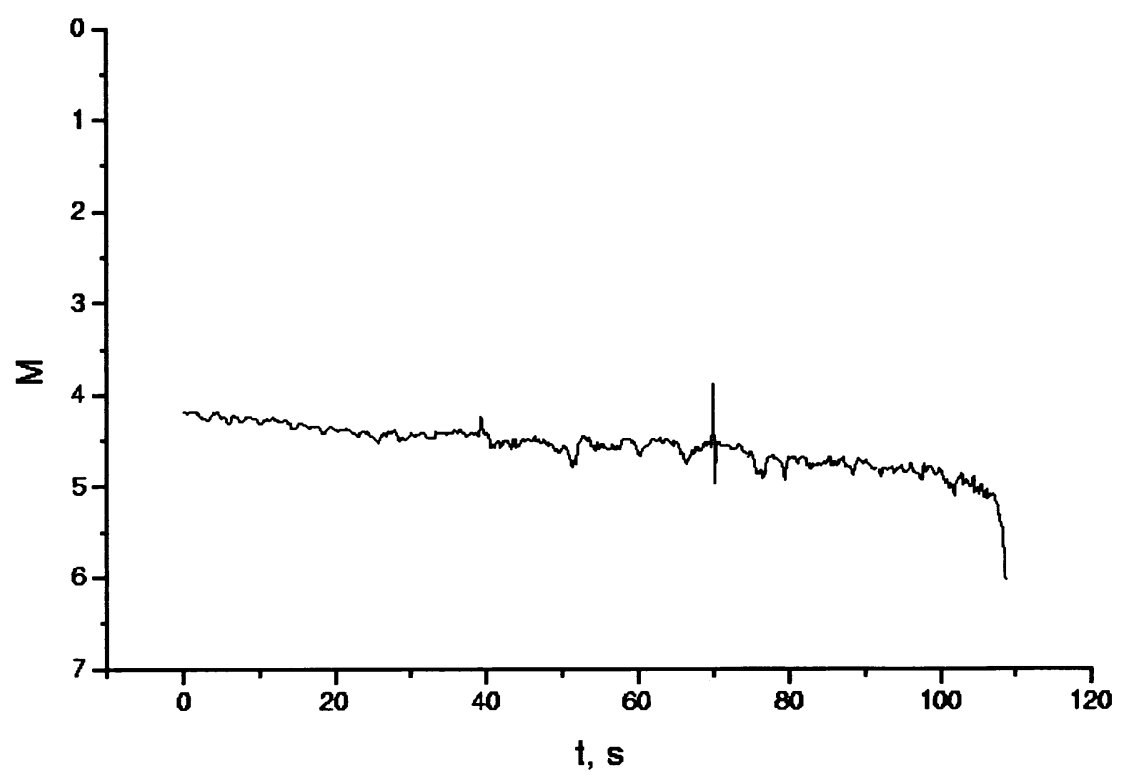

Figure 2. The light-curve of the satellite ERS2 measured on 19th May 1999 at $20 \mathrm{~h} 20 \mathrm{~m} 06,18 \mathrm{~s}$ UT.

The TOPEX was launched in 1992 and is stabilized in space. Hence its lightcurve should be quite smooth with no changes. Fig.2 shows the light-curve of the satellite ERS2, which is stabilized in space. This light-curve was received on the 19th May 1999 at $20 \mathrm{~h} 20 \mathrm{m06}, 18 \mathrm{~s}$ UT. From the internet (where one can find photographs of many artificial satellites) we know that TOPEX has one solar panel, and hence we conclude that the outburst of light which we see on the light-curve $150 \mathrm{~s}$ after the begining is just the reflection of this panel. There are other outbursts on the light-curve which we will not discuss at present.

Solving the geometrical problem Sun-satellite-observer under the condition that the reflection is from the mirror could give the position in space of the panel reflecting the light. Taking into account that on the light-curves one can see many different changes of light, and solving the different problems for each change, one can calculate the position of each detail and then the whole object. That is very simple example of how one can use the light-curve for solving the problem of defining the orientation of the satellite in space.

\section{References}

Flury, W. 1991, Adv.Space Res. v.II, N 12, 67-79

Blagodyr, Ja.T. et al. 1996, Turkish Journal of Physics 20, 879-882 